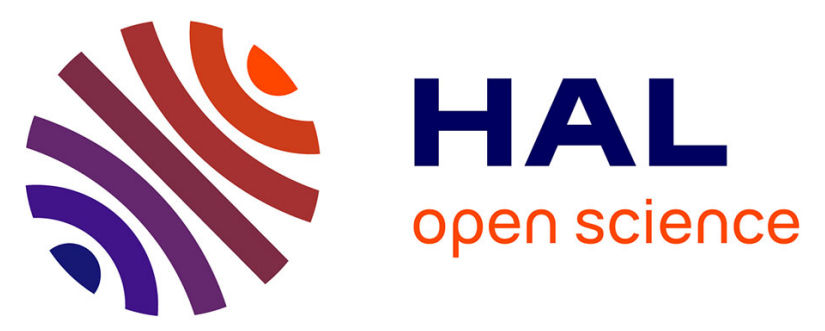

\title{
Nanoscale Switching of Near-Infrared Hot Spots in Plasmonic Oligomers Probed by Two-Photon Absorption in Photopolymers
}

Yinping Zhang, Guillaume Demesy, Mohamed Haggui, Davy Gérard, Jeremie Béal, Stephanie Dodson, Qihua Xiong, Jérôme Plain, Nicolas Bonod, Renaud Bachelot

\section{To cite this version:}

Yinping Zhang, Guillaume Demesy, Mohamed Haggui, Davy Gérard, Jeremie Béal, et al.. Nanoscale Switching of Near-Infrared Hot Spots in Plasmonic Oligomers Probed by Two-Photon Absorption in Photopolymers. ACS photonics, 2018, 5 (3), pp.918-928. 10.1021/acsphotonics.7b01164 . hal01765682

\section{HAL Id: hal-01765682 \\ https://hal.science/hal-01765682}

Submitted on 19 Jul 2018

HAL is a multi-disciplinary open access archive for the deposit and dissemination of scientific research documents, whether they are published or not. The documents may come from teaching and research institutions in France or abroad, or from public or private research centers.
L'archive ouverte pluridisciplinaire HAL, est destinée au dépôt et à la diffusion de documents scientifiques de niveau recherche, publiés ou non, émanant des établissements d'enseignement et de recherche français ou étrangers, des laboratoires publics ou privés. 


\section{Nanoscale switching of near-infrared hot spots in plasmonic oligomers probed by}

\section{two-photon absorption in photopolymers}

Yinping Zhang£, Guillaume Demesy $\$$, Mohamed Haggui ${ }^{£, \&}$, Davy Gérard ${ }^{£}$, Jérémie Béal ${ }^{£}$, Stephanie Dodson\%, Qihua Xiong\%,\#, Jérome Plain ${ }^{£}$, Nicolas Bonod ${ }^{\$}$ and Renaud Bachelot ,** $^{*}$

$£$ Institut Charles Delaunay CNRS UMR 6281, Laboratoire of Nanotechnologie, Instrumentation et Optique (LNIO). Université de Technologie de Troyes, France \$Aix Marseille Univ., CNRS, Centrale Marseille, Institut Fresnel, Marseille, France

${ }^{\%}$ Division of Physics and Applied Physics, School of Physical and Mathematical Sciences, Nanyang Technological University, Singapore 637371

${ }^{\#}$ School of Materials Science and Engineering and § Division of Microelectronics, School of Electrical and Electronic Engineering, Nanyang Technological University, Singapore 639798

*Corresponding author: renaud.bachelot@utt.fr

\& Current address: Institute für Experimentalphysik, Freie Universität Berlin, Berlin 14195, Germany

*Corresponding author: renaud.bachelot@utt.fr

\& Current address: Institute für Experimentalphysik, Freie Universität Berlin, Berlin 14195, Germany

Abstract: Plasmonic oligomers are near-field coupled assemblies of metallic nanoparticles. Both their scattering/absorption spectra and the spatial distribution of the electromagnetic field can be tailored through the hybridization of plasmonic modes hosted by individual particles. Such a control on the field distribution opens new routes to deliver light at a deep subwavelength scale in targeted locations ("hot spots"). However, active control of hot spots in plasmonic oligomers and their observation in the near field are highly challenging. Here, we propose to use a two-photon absorption process in azopolymer in the near infrared to imprint from the far field the near field distribution around a trimer antenna. The trimer antenna comprises two nanogaps separated by a quarter of the wavelength in the polymer and is designed to allow for the switch on a single nanogap when illuminated at $900 \mathrm{~nm}$ by a 
collimated beam at an oblique incidence. The monitoring of the topographical depletions in the photopolymer proves that it is possible to address a single hot spot in the structure and to remotely switch its location in the two nanogaps on demand, simply by illuminating with an opposite oblique incidence. This work shows that bonding and anti-bonding gap modes can be selectively excited resulting in controlled hot spot locations. Two-photon absorption by azobenzene-containing photopolymer turns out to be a reliable approach for investigating confined plasmonic fields in the near infrared with a $20 \mathrm{~nm}$ resolution.

Key words: nanoplasmonics, nanoantenna, molecular probing, 2-photon isomerization, azopolymer 
Metallic nanostructures offer the possibility to strongly confine light at nanometer scale. ${ }^{1-5}$ In general, rational control of the plasmonic nanostructures' geometry and/or incident beam shaping (including phase, polarization state and angle of incidence) can produce a large variety of near-field distributions that can be exploited in photonic computing, ${ }^{6,7}$ nanophotochemistry, ${ }^{8-11}$ optical trapping, ${ }^{12-15}$ non-linear nanoptics, ${ }^{16-18}$ Surface Enhanced Raman Spectroscopy, ${ }^{19-21}$ plasmon-assisted light emission (including single photon emission), ${ }^{22-28}$ and hot carrier generation and collection. ${ }^{29-31}$

In this context, plasmonic oligomers are very interesting to efficiently confine and enhance light intensity at deep subwavelength scale within the gaps separating the different plasmonic particles. ${ }^{32-35}$ The so-called hot-spots result from the coupling between the localized surface plasmon modes hosted by the individual particles. Multiple hot-spots can be created simultaneously in plasmonic oligomers and their remote control remains challenging.

Trimers of gold nanorods are examples of oligomers that are highly suited to yield strong field enhancements. ${ }^{36-38}$ One of the challenges is to switch on or switch off on demand light in the two available gaps. For incident polarization parallel to the trimer axis, the plasmonic bright hot spots result from the excitation of a bonding mode, i.e. when the dipolar moments in the neighboring particles are in phase, while the anti-bonding modes (out-of phase adjacent dipolar moments) are dark. It was first proposed to control the hot spots by tailoring the phase of the incident beam. G. Volpe et al. used Laguerre Gaussian beams to shape the phase of the incident beam and managed to excite anti-symmetric bonding modes in the two particles associated with a nanogap and a symmetric anti-bonding mode in the two particles associated with the other nanogap. ${ }^{39}$ Far-field observation based on two-photon induced luminescence supported this approach. A simpler strategy was proposed by Devilez et al., consisting in the simultaneous excitation of the symmetric and anti-symmetric modes in the trimers without shaping the phase of the incident beam. ${ }^{40}$ They considered a simple plane wave excitation and showed theoretically that by simply tilting the incidence, it is possible to excite this combination of modes, corresponding to a subradiant mode. The near-field intensity can be almost perfectly vanished in a given gap while strongly enhanced in the other gap, even if they are separated by only a tenth of the incident wavelength. By illuminating the trimer antenna under the opposite incidence angle, the hot spot is switched in the other nanogap. Hence, the position of the electromagnetic hot spot can be controlled at deep 
subwavelength scale by a simple far-field optical command. This effect is highly promising because it would easily enable rational control of nanometric hot spots for many applications such as those cited at the beginning of the introduction.

Experimental observation and study of this effect at the nanoscale are crucial for its control and optimization. In practice, the near-field investigation of resonant plasmonic nanogaps constitutes a challenging task for several reasons. For example, the use of Scanning Near-field Optical Microscopy (SNOM) ${ }^{41}$ is complicated by the presence of the probe, modifying the resonance conditions for hot spot excitation: the observed resonance would mainly result from the coupling between gap modes and the probe, rather than the optical properties of the plasmonic cavity itself. ${ }^{42}$ Additionally, both the nature of the tip-sample optical interaction and the interpretation of the SNOM images ${ }^{43}$ strongly depend on the probe (shape, type, quality, control mode,...) and its environenement, ${ }^{44,45}$ which is pretty difficult to control. Other methods were recently reported to study plasmonic nanostructures at the nanoscale. Photon Emission Electron Microscopy (PEEM), ${ }^{46-48}$ based on the photoelectrical effect, has turned out to be highly efficient but requires an electron reservoir. As a result, near-field contrast can be observed only on metal surface and not at zones far from metal. A gap-mode field that is partly delocalized in the dielectric gap medium is not expected to lead to any easily interpretable PEEM contrast, despite some interesting reported data. Additionally, because of the out-of plane electric field used for electron accelerations, this technique is mainly sensitive to out-of plane field components whereas local fields that result from inplane plasmonic coupling are mainly in-plane polarized. This important point will be discussed further. As far as Electron Energy Loss Spectroscopy (EELS) is concerned, ${ }^{49-54}$ contrasts result from local electronic absorption (a metal reservoir is thus also required) and no optical excitation is possible (excitation is purely electronic in EELS). Additionally, it was recently shown that inside the gap region of a coupled plasmonic system, the EELS signal can become zero for the bonding mode and maximal for the antibonding mode. ${ }^{53-54}$ In general, PEEM and EELS techniques are very efficient but pretty sophisticated and expensive.

Here, we report on the direct near-field observation, based on a simple method, of mode balancing and localization of hot spots in a trimer plasmonic nanoantenna. We used twophoton absorption in Photosensitive Azobenzene-containing Polymer (PAP) for plasmonic nano-imaging of infrared nanoantenna, opening the route to near-field investigation of coupled systems based on molecular probing.

The linear plasmonic trimer antenna under study is presented in Figure 1. It was made by electron-beam lithography and consists of a trimer of gold nanorods (115 nm long, $45 \mathrm{~nm}$ 
wide, and $50 \mathrm{~nm}$ high) separated by two $30 \mathrm{~nm}$ wide gaps denoted C1 and C2 separated by a $145 \mathrm{~nm}$ distance, i.e. $\lambda / 4\left(\lambda=\lambda_{0} / \mathrm{n}\right.$, where $\lambda_{\mathrm{o}}$ is the incident wavelength and $\mathrm{n}$ is the PAP refractive index). Figure 1(a) shows an image of such a trimer as observed by scanning electron microscopy (SEM).

(a)

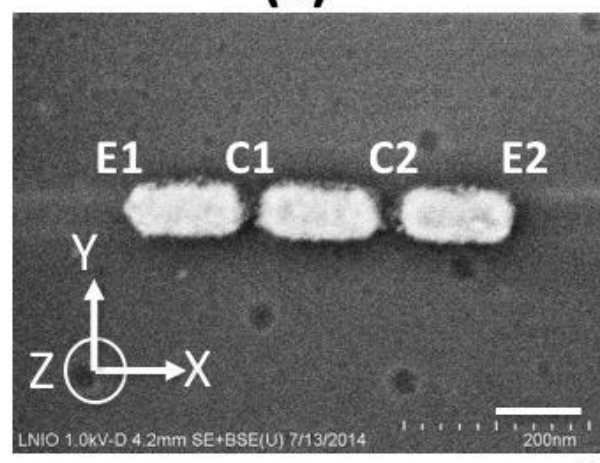

(b)

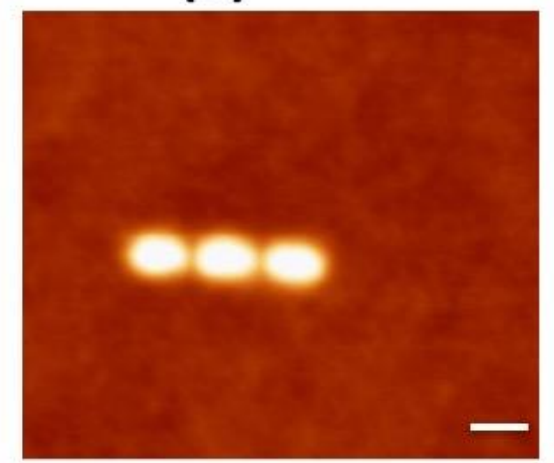

(c)
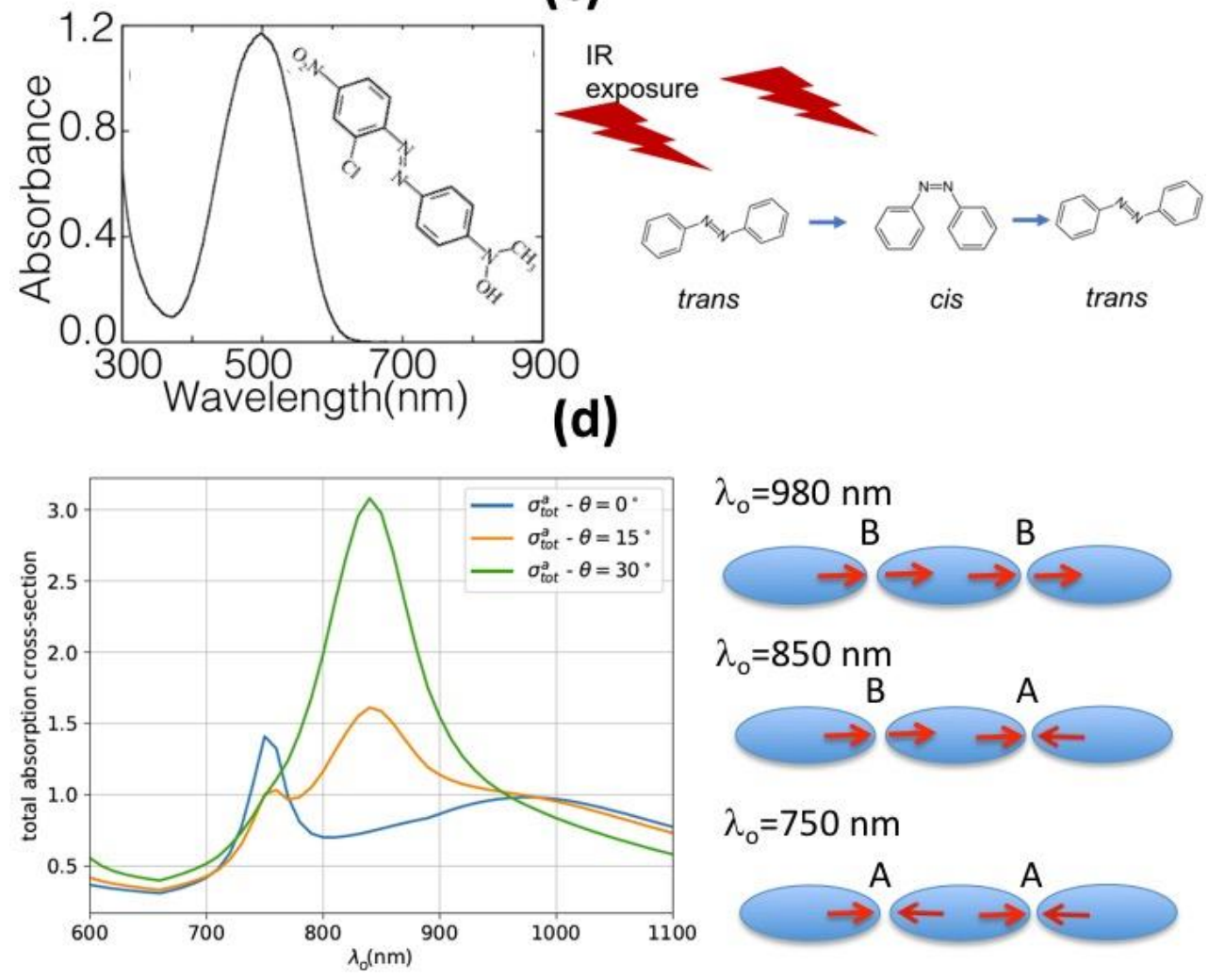

$\lambda_{0}=980 \mathrm{~nm}$

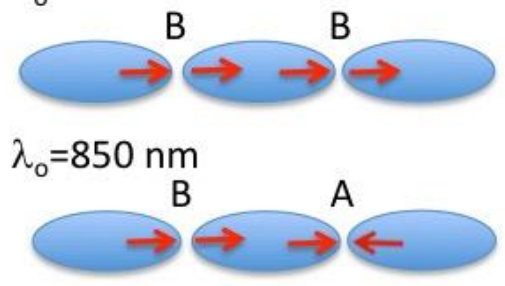

$\lambda_{0}=750 \mathrm{~nm}$

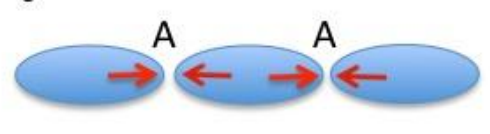

Figure 1. Plasmonic trimer and molecular probe. (a) SEM image of the studied trimer. White scale bar represents $100 \mathrm{~nm}$. (b) Atomic Force Microscope (AFM) image of a gold trimer covered with a 45 $\mathrm{nm}$-thick layer of azobenzene-containing photosensitive copolymer. Apparent height is about $15 \mathrm{~nm}$. White scale bar represents $100 \mathrm{~nm}$. AFM profiles will be shown in the following. (c) Azobenzene molecule used as a nanomotor for near-field probing (see text for details). Left: absorption spectrum and representation of the active molecule. Right: principle of the molecular motion based on 2-photon 
isomerization. (d) Left: Calculated absorption spectrum of the trimer (realistic structure) for different incident angles. Right: representation of the different states of vibration of charges at different resonant wavelengths.

Our main motivation is to experimentally demonstrate that it is possible to switch on and off, on demand, the respective near-field enhancement at each cavity ( $\mathrm{C} 1$ or $\mathrm{C} 2)$. We will also pay attention to the extremities E1 and E2 depicted in Figure 1(a), as they can support electromagnetic hot spots as well. The left-hand side of Figure 1(d) shows calculated (see method) absorption spectra of the trimer antenna on glass and coated with a $45 \mathrm{~nm}$-thick PAP film when illuminated by an unpolarized plane wave propagating along the $\mathrm{Z}$ axis (illustrated in Figure 1(a)) for different angles of incidence $\theta$. The absorption spectrum shows a strong optical response in the near-infrared. First, we can observe the strong dependence of the spectrum with respect to the angle of incidence. In normal incidence $\left(\theta=0^{\circ}\right)$, the absorption spectrum features a peak around $\lambda_{0}=980 \mathrm{~nm}$ due to the excitation of the radiant (bright) mode. This mode corresponds to a situation where the electric dipolar moments associated with each nanoparticle are aligned and in phase, resulting in two gap bonding modes, denoted B-B (see first illustration to the right of the spectrum). To this low absorption is associated a strong scattering efficiency (see supplementary information). One can also observe a secondary peak at $750 \mathrm{~nm}$ due to the excitation of the mode for which the dipolar moments are out of phase, resulting in two anti-bonding gap modes, denoted A-A (see third illustration to the right of the spectrum). It is much more pronounced in the absorption spectrum and can be called "dark mode", corresponding to the excitation of a high-order resonance in the nanoantenna. In oblique incidence, with a moderate angle of incidence $\left(\theta=15^{\circ}\right)$, three peaks can be observed. The radiant and dark modes can again be observed at $\lambda_{0}=980 \mathrm{~nm}$ and $\lambda_{0}=750 \mathrm{~nm}$ respectively, while a subradiant mode is excited at $\lambda_{0}=850 \mathrm{~nm}$. This mode, described for example by $\mathrm{F}$. Frimer et al. ${ }^{55}$ and Mirin et al., ${ }^{56}$ corresponds to both in-phase and opposite-phase pairs of dipoles resulting in both anti-bonding and bonding gap modes within the trimer (denoted A-B 
or B-A, see second illustration to the right of the spectrum). It should be pointed out that A-B and B-A are degenerate modes. When the angle of incidence is increased, the component of the electric field along the trimer axis weakens leading to the cancellation of the radiant mode while the subradiant mode is still excited. Figure 1(d) shows that the gold trimer has strong optical response in the near-infrared. The excitation of this subradiant mode from the far field around $\lambda_{0}=850 \mathrm{~nm}$ in oblique incidence, especially at an incidence of $30^{\circ}$, opens new routes to tailor the distribution of the near electric field in the two gaps of the trimer antenna. More detailed discussion on trimer modal analysis can be found in supporting information. Based on calculated spectrum in Figure 1(d), the incident wavelength of $900 \mathrm{~nm}$ was chosen, in order to be sensitive to both radiant and subradiant modes. We will see later that this wavelength is also suitable for 2-photon absorption in PAP used for near-field probing.

To visualize the optical near-field, we used a specific approach that has been developed over the past decade. ${ }^{57-65}$ Azobenzene molecules (see Figure 1(c)) that are covalently attached to a polymeric backbone are used as optically driven molecular nanomotors. Light can excite the trans $\leftarrow \rightarrow$ cis isomerization in azobenzene-like molecules that are grafted to chains of polymethyl methacrylate (PMMA). This copolymer is usually named PMMA-Dispersed red one (PMMA-DR1) because it looks red since its absorbance is maximum in the range 400$500 \mathrm{~nm}$ (see the left-hand side of Figure 1(c)). Upon absorbing a photon in the visible, an isomerization cycle produces a "worm-like" motion that leads to a displacement along the transition moment axis that could be on the order of a nanometer (see right-hand side of Figure 1(c). ${ }^{66}$ Many repeated isomerization cycles ultimately lead to more large-scale mass transport. After exposure to light, an Atomic Force Microscope (AFM) is used to measure the resulting topography that is related to the near-field intensity pattern to be characterized. The azobenzene-like molecules are thus nanomotors that push and pull at the polymer material 
leading to topographical features after exposure that reflect the underlying near-field intensities. As it will be seen, this method is also sensitive to local field polarization. More details about this optical near-field imaging method in the visible can be found in references. ${ }^{57-63}$ In particular, in reference ${ }^{60}$ we theoretically described optically-induced topographical formation based on a multiscale statistical approach.

Figure 1(b) shows an AFM image of a trimer antenna covered by a $45 \mathrm{~nm}$-thick layer of PMMA-DR1 for near-field investigation through mass transport. The typical absorption spectrum of the co-polymer is in the (400-600) nm range (see Figure 1(c)). This feature is the raison why all the near-field studies have been so far done in the visible, which is not relevant for gap mode study because plasmonic couplings generally result in red-shifted plasmon resonance, making a priori impossible the study of the resonant nanoantenna in the infrared.

In order to address this important issue, we propose to follow a two-photon isomerization, which allows us to illuminate the antenna in the near infrared spectrum.
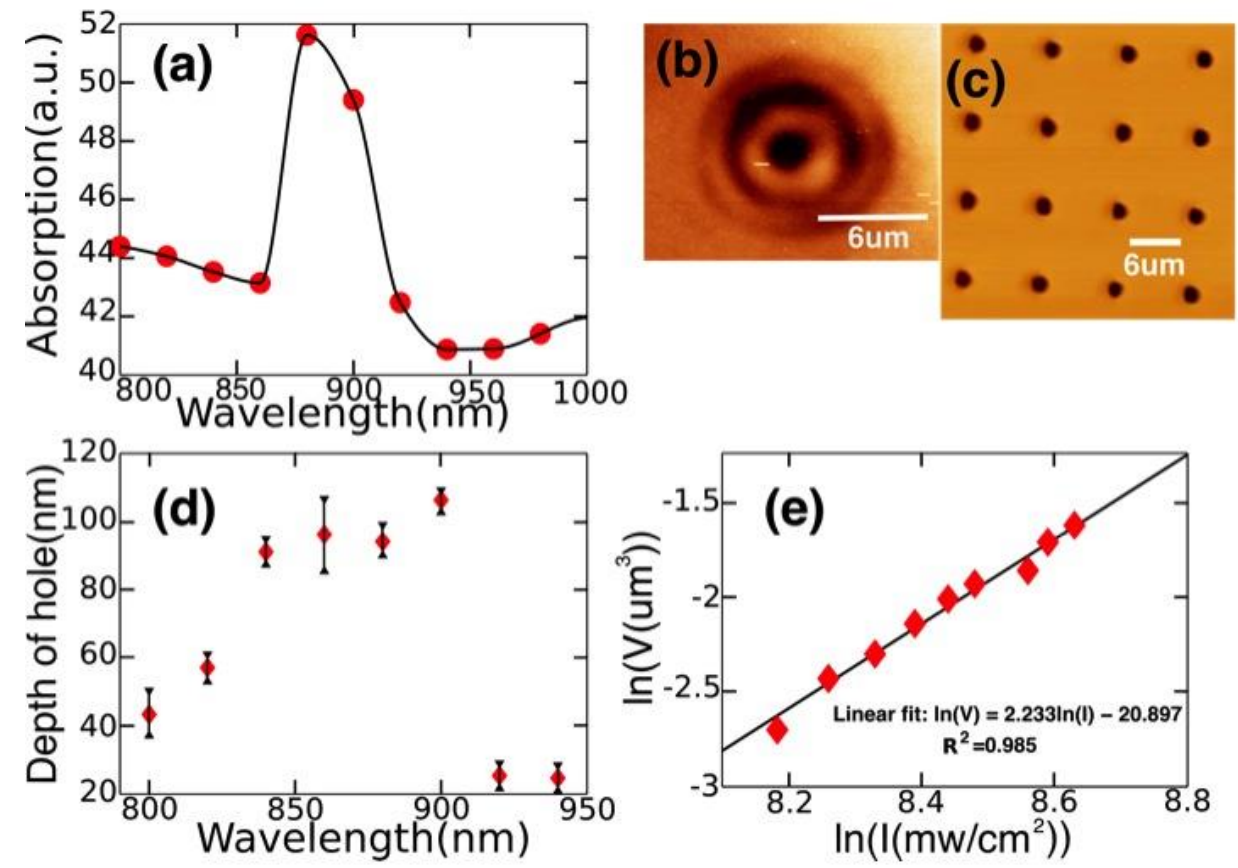

Figure 2. Two-photon absorption of PMMA-DR1. (a) IR absorption spectrum of a $100 \mathrm{~nm}$ thick layer of PMMA-DR1 obtained by using a tunable Ti:Sa laser. (b) Induced topography (AFM image) in the case of a focused beam at a $900 \mathrm{~nm}$ wavelength. (c) Two-photon scanning optical lithography on PMMA-DR1. (d) Depth of holes as a function of the wavelength. (e) Hole volume as a function of the incident power density (log-log plot). 
We start this study by characterizing the two-photon absorption of a $100 \mathrm{~nm}$ thick monolayer of PMMA-DR1 coated on a glass substrate. Figure 2(a) shows the absorption spectrum of the monolayer. A strong absorption peak is observed around $900 \mathrm{~nm}$, indicating two-photon absorption in the copolymer film. Figures 2(b) and 2(c) demonstrate that this absorption can result in matter displacement, leading to a clear depletion in the film. In Figure 2(b), we use a laser beam $\left(\lambda_{0}=900 \mathrm{~nm}\right)$ focused with an objective lens $(\mathrm{N} . \mathrm{A}=0.3)$ to print an Airy pattern whose central peak results in a deep depletion. Based on the use of this central depletion, a proper exposure time and intensity can enable scanning lithography of PMMA-DR1, as illustrated in Figure 2(c). Many published works have discussed the origin of this depletion. In particular, it was shown that molecules escape from in-plane polarized light with an inplane motion, resulting in a topography that is the negative image of the intensity distribution on in-plane fields ${ }^{57}$ Figure $2(\mathrm{~d})$ illustrates another signature of the spectral sensitivity of the copolymer in the infrared. Hole depths observed in Figure 2(c) were measured as a function of the wavelength for a fixed power, revealing a clear sensitivity around $900 \mathrm{~nm}$. The twophoton nature of the absorption was confirmed by studying the depletion volume of the holes as a function of the incident power. The resulting $\ln$ - $\ln$ plot (Figure 2(e)) presents a slope of 2, which is the signature of the quadratic optical response characteristic of two-photon absorption.

Although 2-photon induced movement in PAP has already been reported, ${ }^{67}$ we used for the first time two-photon absorption in PMMA-DR1 polymer to study the near fields on plasmonic antennas in the near-infrared.

Figure 3 depicts the experimental configuration for near-field investigations of linear plasmonic trimer antennas. A 45-nm thick PMMA-DR1 layer was deposited on the sample by spin coating (see method), resulting in an effective $10 \mathrm{~nm}$ thickness at the top on the gold particles and a $45 \mathrm{~nm}$-thickness elsewhere. The apparent height of the particles is thus about $15 \mathrm{~nm}$ after spin coating. Figure 1(b) is the AFM image of the PMMA-DR1-coated antenna 
before exposure. In the following, such an image will not be shown again and all presented photo-induced topographies should be compared to it because each exposure was done from a plasmonic structure covered by a fresh layer of unexposed PMMA-DR1, as illustrated in Figure 1 (b). AFM cross-sections taken before and after exposure will be shown for comparison.

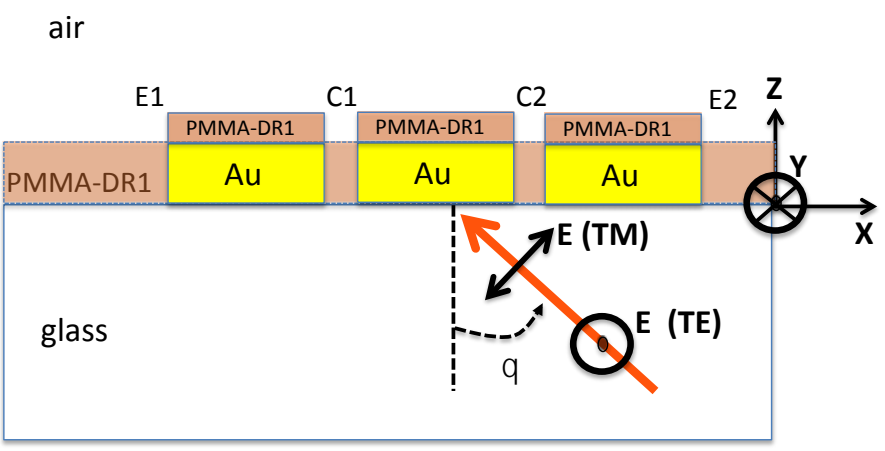

Figure 3. Experimental configuration used for near-field imaging of gold trimers. $900 \mathrm{~nm}$ wavelength was used (see text for details).

The incident light consists of a slightly focused linearly polarized beam (plane wave) from a Ti:Sa laser emitting at $900 \mathrm{~nm}$ (see the above discussion). This wavelength turned out to be relevant for both excitations of the subradiant/radiant mode and two-photon absorption of PMMA-DR1.

Average power density and exposure time were $300 \mathrm{~mW} / \mathrm{cm}^{2}$ and $60 \mathrm{~s}$ respectively. Both incidence angle $(\theta)$ and electric field direction (Transverse Electric, TE, or Transverse Magnetic, TM) constitute two important parameters that allow for a far field control of the hot spot switch in the antenna.

Due to experimental constraints, possible fluctuations of both the antenna's geometry and the polymer thickness can be observed. Typical fluctuations are in the 0-15\% range. Four specific locations are pointed out in Figure 3: the two nanogaps $(\mathrm{C} 1$ and $\mathrm{C} 2)$ and the two extremities (E1 and E2). We will see that the (E1, C1, C2, E2) set can constitute a tunable four-digit nanosystem. 
Figure 4 shows the result of the exposure in the TE case ( $\mathrm{Y}$ incident polarization) at normal incidence $\left(\theta=0^{\circ}\right.$, except for Figure 4(d)). Figure 4(a) shows the photoinduced topography after exposure, as observed by Atomic Force Microscopy (AFM). The Y oriented incident field coupled with plasmon oscillations occurred transversally to the main axis of the oligomer,, resulting in negligible couplings between the individual nanoparticles along the $\mathrm{X}$ axis. As a result, the near-field dipole radiation, parallel to the incident field, of each individual particle is revealed by AFM, as compared to the AFM image of the same structure before exposure (Figure 1(b)). The apparent photoinduced matter depletion results from the vectorial sensitivity of molecular displacement, as the vectorial nature of the near-field has to be taken into account for proper interpretation of the photoinduced topography. ${ }^{63}$
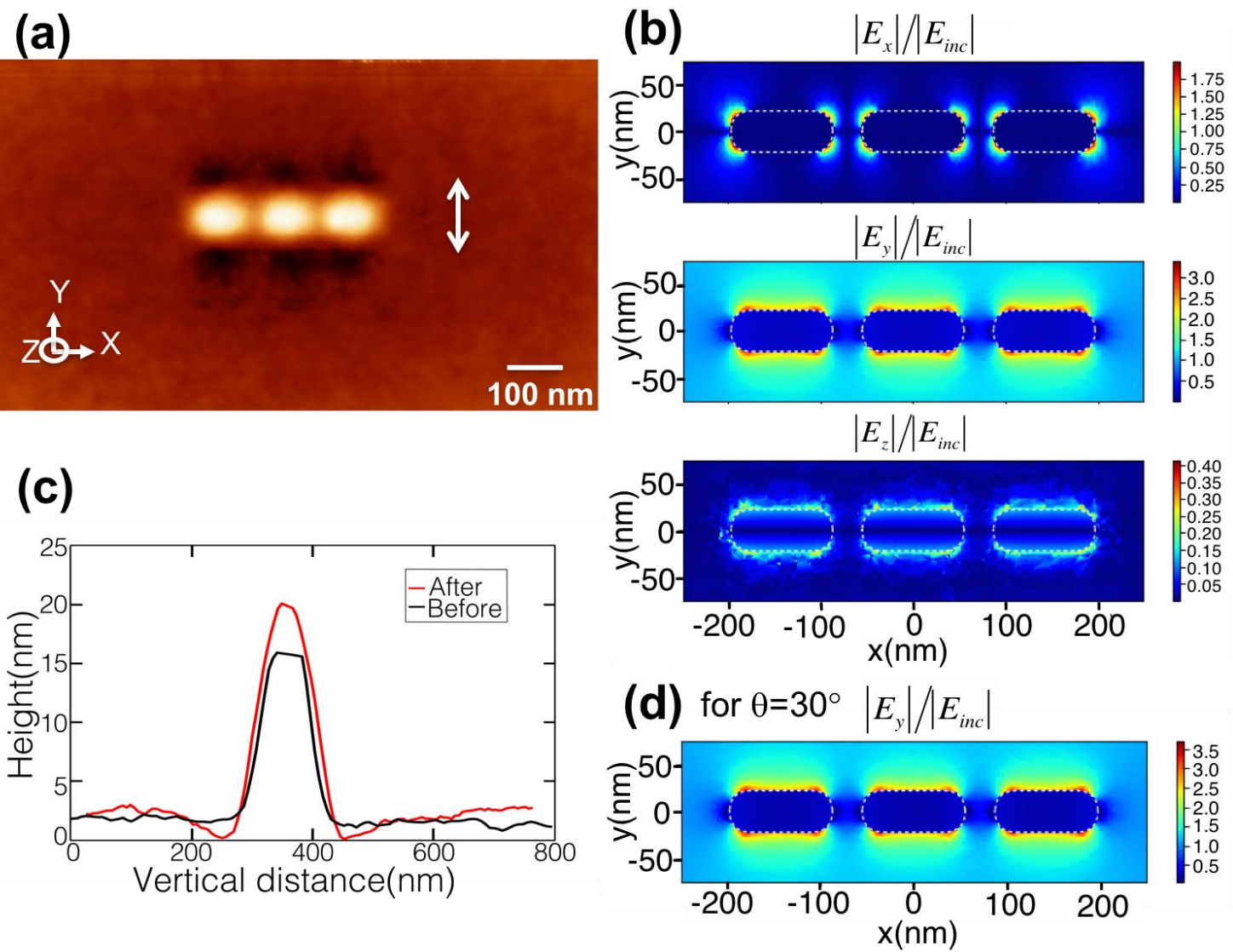

Figure 4. Result of the exposure for TE polarization at normal incidence $\left(\theta=0^{\circ}\right.$ except for calculated Figure 4(d) for which $\theta=30^{\circ}$. Exposure wavelength is $900 \mathrm{~nm}$. (a) AFM image after exposure. (b) Distribution of absolute value of each field component calculated with Finite Element Method (FEM). (c) Y cross sections above one particle, taken before and after exposure. In all the cross sections throughout the article, height scale was set so that 0 
corresponds to the lowest topography and height range is fully covered. (d) Absolute value of the Ey field component for $\theta=30^{\circ}$ showing no influence of the incident angle.

Figure 4(b) shows the calculated absolute value of each field component for a realistic PMMA-DR1-coated antenna illuminated at normal incidence with a Y polarization $\left(\lambda_{0}=900\right.$ $\mathrm{nm})$. It shows pretty weak $\mathrm{Y} \rightarrow \mathrm{X}$ and $\mathrm{Y} \rightarrow \mathrm{Z}$ depolarization. In the case of TE polarization, the plasmon field remains mainly Y polarized: matter principally moved away from light parallel to the involved near-field component leading to a topographical depletion at high intensity zones. This is why Figure 4(a) mainly looks like the negative image of calculated $E_{y}$ map of Figure 4(b). Migrated matter partly accumulated at the top of particles as shown in the crosssection in Figure 4(c). In other words, Figure 4(a) is a near-field snapshot of the individual Y polarized near-dipole radiation of each particle of the trimer. Figure 4(d) shows the calculated absolute value of the $\mathrm{E}_{\mathrm{y}}$ component mainly involved for oblique incidence $\left(\theta=30^{\circ}\right)$. It is worth noticing that the angle of incidence has no significant effect on the field distribution making clear that TE polarization is not suitable for tunable mode balancing. It is worth pointing out that the PEEM technique would have been mainly sensitive to the $E_{z}$ field map shown in Figure $4(b)$, to the detriment of $E_{y}$ (that characterizes best the trimer optical response for $\mathrm{Y}$ polarized excitation), for two main raisons: i) photoelectrons are accelerated along $\mathrm{Z}$ direction in PEEM and ii) $E_{y}$ is partly delocalized from metal surface from where electrons are ejected. 


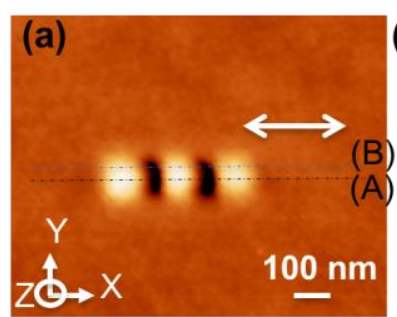

(d)

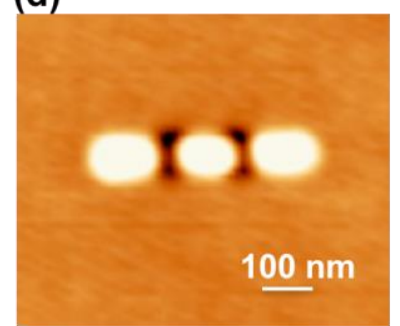

(e)

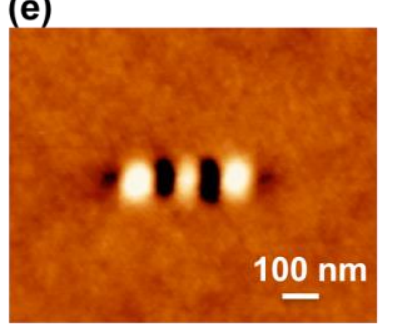

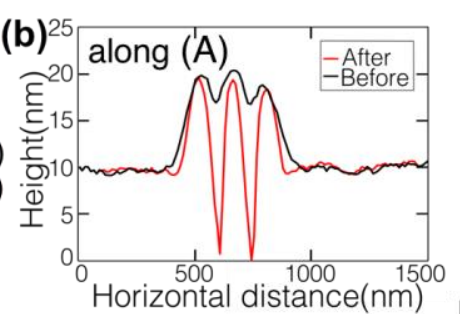

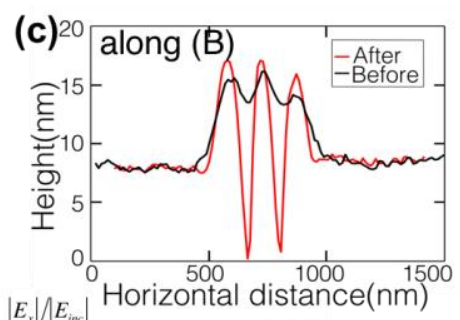

(f)
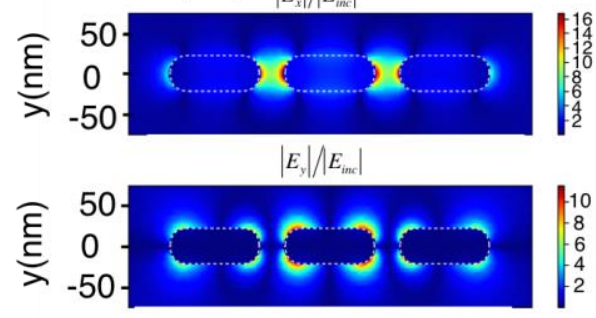

$\left|E_{z}\right| /\left|E_{\text {inc }}\right|$

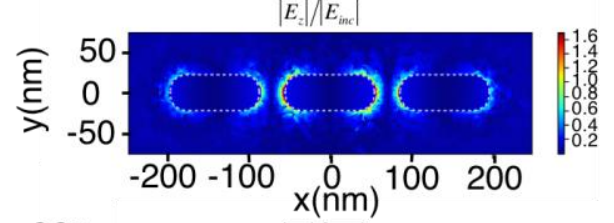

(g) for $\theta=30^{\circ}$

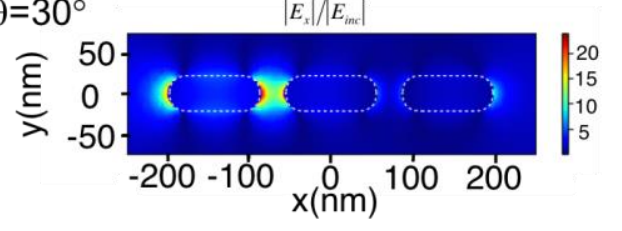

Figure 5. Result of the exposure for TM polarization at normal incidence $\left(\theta=0^{\circ}\right.$ except for calculated Figure $5(\mathrm{~g})$ for which $\theta=30^{\circ}$. Exposure wavelength is $900 \mathrm{~nm}$. (a) AFM image after exposure. $\left.\mathrm{b}, \mathrm{c}\right) \mathrm{X}$ Cross sections above the trimer, taken along the axes (A) and (B) respectively. (A) and (B) are shown in Figure 5(a). (d) AFM image taken after weaker intensity exposure. (e) AFM image taken after higher intensity exposure. (f) Calculated absolute value of each component. (g) Calculated absolute value of Ex field for $\theta=30^{\circ}$ showing the influence of the incident angle.

Figure 5 shows the result of the exposure in the TM case (incident polarization parallel to the main axis of the nanoantenna trimer) at normal incidence (except for Figure 5(g)). The photoinduced topography after exposure displayed in Figure 5(a) confirms that the two hot spots at $\mathrm{C} 1$ and $\mathrm{C} 2$ are excited. In particular, the field distribution is no longer strictly symmetric relatively to each single gold particle and E1, E2 antenna extremities are not (or poorly) excited. Figure 5(a) is a near-field map of the radiant B-B mode described in Figure 1(d). Figure 5(f) shows the calculated absolute value of each field component. Again, it shows pretty weak $X \rightarrow Y$ and very weak $X \rightarrow Z$ depolarization. In the case of a TM excitation, the plasmon field is localized within the gaps and mainly $\mathrm{X}$ polarized: photo-activated matter 
principally moved parallel to the involved near-field leading to a topographical depletion at high intensity zones. This depletion is shown in Figure 5(b): matter migrated from cavities C1 and $\mathrm{C} 2$ and was partly accumulated at the top of particles, at the edges of the trimer, as shown in Figure 5(c). In other words, Figure 5(a) is, at the first approximation, a snapshot of the Xpolarized near-field coupling in the plasmonic trimer. However, it turns out that Figure 5(a) hides some features that are the signature of weak Y-polarized near-fields. Weaker exposure (300 $\mathrm{mW} / \mathrm{cm}^{2}$ for $30 \mathrm{~s}$.) led to the optically induced topography shown in Figure 5(d). This topography presents features that are the signature of Y-polarized field map (see second calculated image of Figure 5(f)). On the other hand, for higher exposure dose $\left(600 \mathrm{~mW} / \mathrm{cm}^{2}\right.$ for 60 s.) $E_{x}$ dominates (see Figure 5(e)) and even local field enhancement at the trimer extremities (E1, E2) are visible. We explain this behavior by assuming that molecular motion along $\mathrm{Y}$ is easier due to free space requirement: ${ }^{58}$ at low intensity, probability of molecular absorption is very weak for both $\mathrm{E}_{\mathrm{x}}$ and $\mathrm{E}_{\mathrm{y}}$ fields. As there is free space along the $\mathrm{Y}$ direction (no $\mathrm{Au}$ particles are expected to block this motion), only $\mathrm{E}_{\mathrm{y}}$ leads to molecular motion, resulting in Figure 5(d). At higher dose, the influence of $E_{x}$ becomes visible and the final topography looks like $\mathrm{E}_{\mathrm{x}}$ distribution, masking the contribution of $\mathrm{E}_{\mathrm{y}}$. Again, let us stress that, in principle, PEEM would have been poorly sensitive to the involved $E_{x}, E_{y}$ fields. Figure $5(\mathrm{~g})$ is the calculated absolute value of the $E_{x}$ field at oblique incidence $\left(\theta=30^{\circ}\right)$. Unlike for the TE case, it shows a clear modification of the field distribution: energy is concentrated at gap $\mathrm{C} 1$, to the detriment of $\mathrm{C} 2$, illustrating the possibility of mode balancing in the plasmonic oligomer.

In order to discuss further the experimental observations, calculations were performed at different wavelengths. Figure 6 shows the calculated intensity $I_{1}, I_{2}$ at gaps $C 1$ and $C 2$ (respectively) as a function of the wavelength for different incidence angles $\left(0^{\circ}, 15^{\circ}, 30^{\circ}\right)$ for a TM incident polarization. 


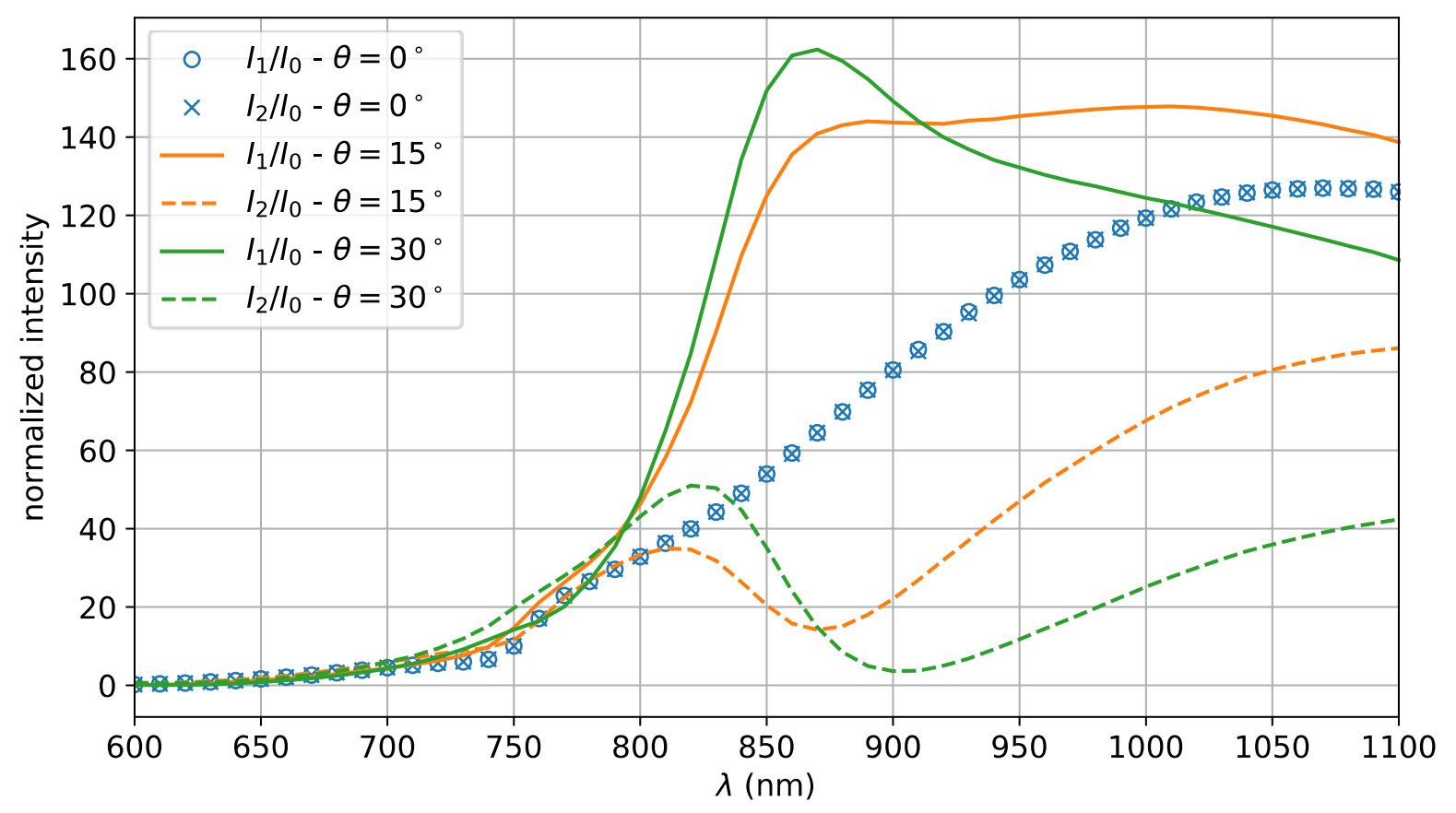

Figure 6. Calculated near-field intensity $\mathrm{I}_{1}$ and $\mathrm{I}_{2}$ at gaps $\mathrm{C} 1$ and $\mathrm{C} 2$ respectively, for different angles of incidence, in the case TM polarization.

In the case of normal incidence $\left(\theta=0^{\circ}\right)$, a clear balance between the two gaps is observed $\left(\mathrm{I}_{1}=\mathrm{I}_{2}\right)$ due to the symmetry of the configuration. This balance is in agreement with the symmetric response observed experimentally in Figures 5(a-c). It is worth noticing, again, that the optical response of the plasmonic trimer is both significant and of interest for wavelengths larger than $800 \mathrm{~nm}$, justifying the use of a $900 \mathrm{~nm}$ wavelength and the exploration of two-photon absorption in azo-polymers. In particular, for $\lambda_{0}>800 \mathrm{~nm}$, a clear distinction can be done between the field of the two nanogaps by tuning the incident angle. The incidence condition at $\theta=30^{\circ}$ and $\square \lambda_{0}=900 \mathrm{~nm}$ is of great interest: almost zero intensity is predicted at $\mathrm{C} 2$ while $\mathrm{C} 1$ presents a strong localized field. This situation corresponds to a B-A subradiant mode described in Figure 1(d). We now aim at observing experimentally this phenomenon (see Figure 7).

The sample was illuminated at $\theta=30^{\circ}$ and $900 \mathrm{~nm}$ in TM polarization, and the observation of the molecular motion in Figures 7(a) and 7(b) reveals that the nanogap C1 was turned on (23 
$\mathrm{nm}$ topographical depletion) while activation of nanogap C2 is very weak ( $3 \mathrm{~nm}$ depletion). This near-observation constitutes an experimental evidence of a B-A subradiant mode, which is in agreement with Figure 6 that predicts a near-zero intensity $I_{2}$ at $C 2$ and an $I_{1}$ intensity (at C1) of about 140. This result shows up the possibility i) to switch on demand and from far field region electromagnetic hot spots at cavities separated by $\lambda_{0} / 4 \mathrm{n}$, ii) to use this effect for remote activation of azobenzene molecules at the nanoscale. It is worth noticing that a secondary weaker hot spot can be identified in Figures 7(a) and 7(b) at the trimer left extremity E1, opposite to the incident direction, which is confirmed by the calculated modulus of the Ex field component in Figure 7(c). Let us now induce the switch of the hot spot location by illuminating the sample with an opposite incidence at $\theta=-30^{\circ}$. Both molecular motion and calculations displayed in Figures 7(d), 7(e), 7(f) show that nanogap C1 is turned off while activation of nanogap C2 is much stronger. This case is clearly symmetrical to the previous one, it corresponds to a A-B subradiant mode. Figures 7(a-f) illustrate well the nanoscale switch between the two gaps.

Figures $7(\mathrm{~g})$ and $7(\mathrm{~h})$ are zoom-out of AFM images in the $\theta=30^{\circ}$ case, taken before and after exposure respectively. Additionally to the near-field contrast, Figure 7(h) exhibits a topographical modulation that corresponds to a near-field diffraction pattern in the Fresnel zone. 
(a)

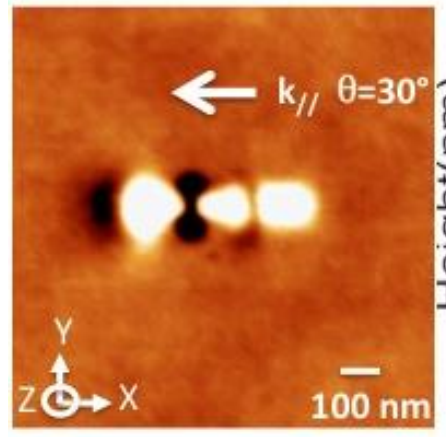

(d)

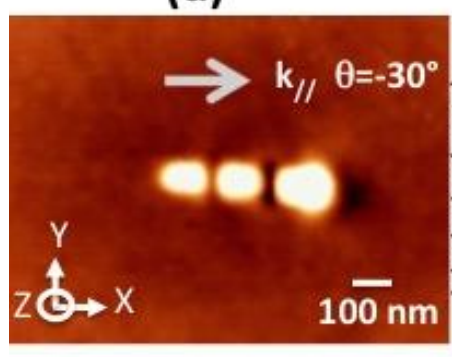

(b)

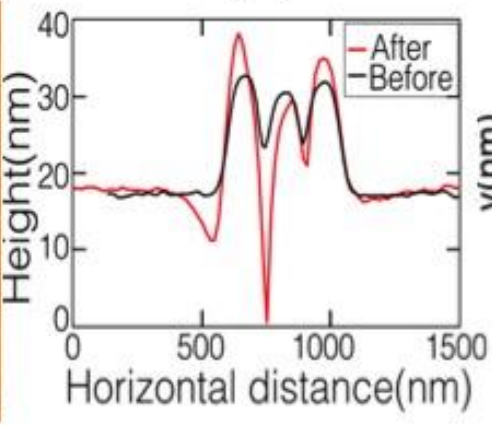

(e)

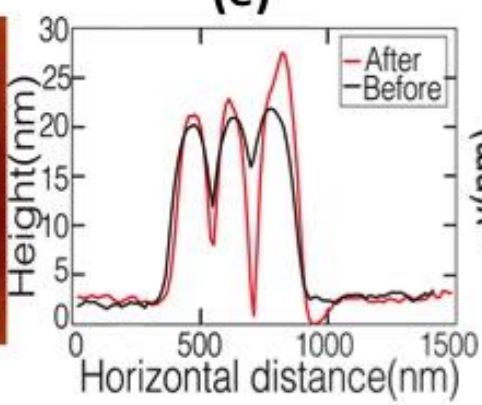

(f)

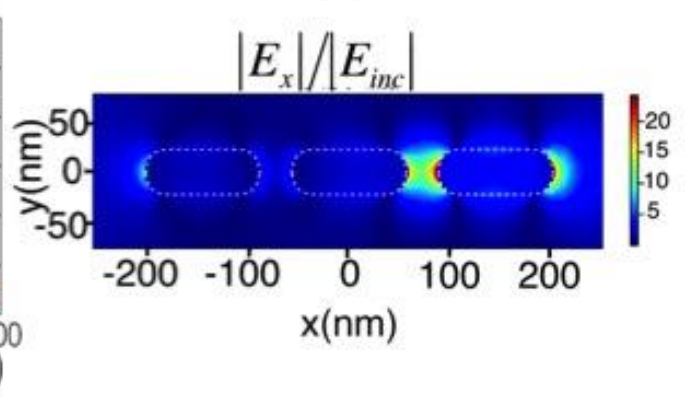

(g)

(h)

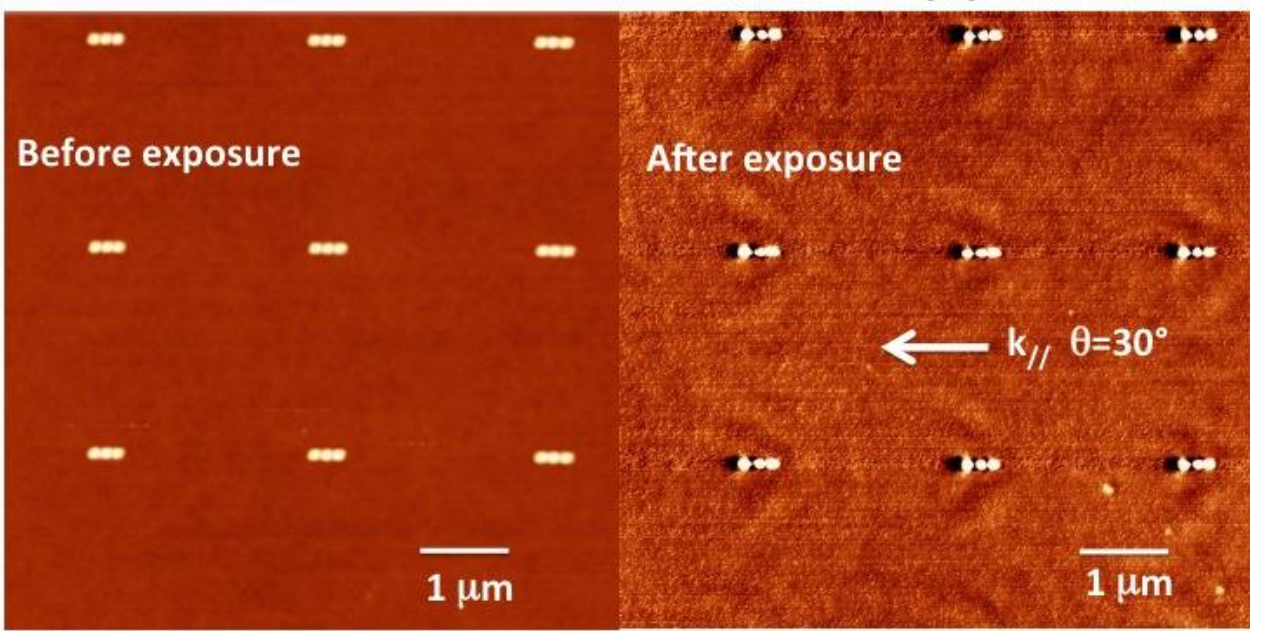

(i)

(j)

(k)
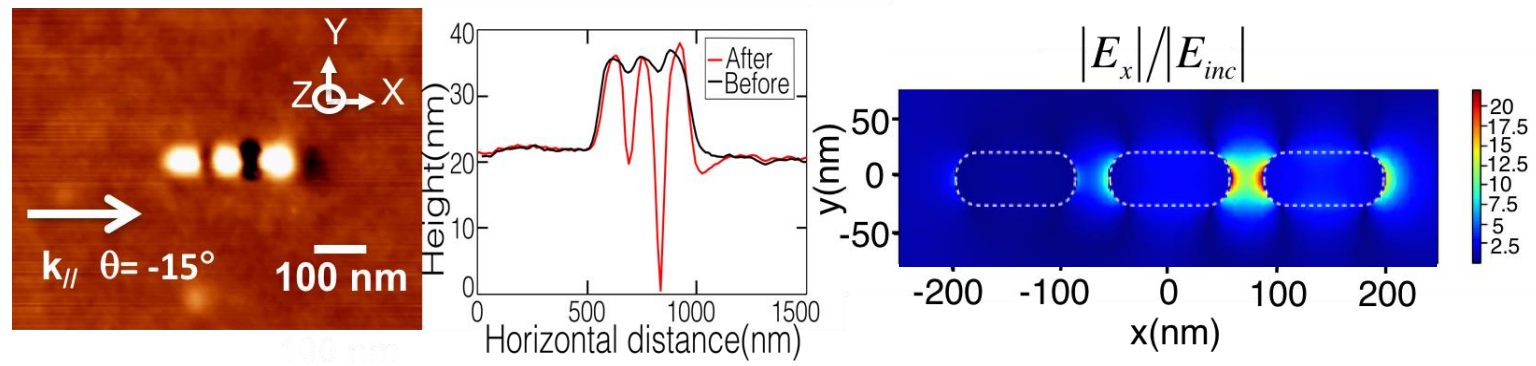

Figure 7. Result of the exposure for TM polarization at oblique incidence. Exposure wavelength is 900 nm. (a),(b),(c) Case of $\theta=30^{\circ}$. (a) AFM image. (b) X Cross sections above the trimer. (c) Calculated map of the absolute value of the Ex field component. (d),(e),(f) Case of $\theta=-15^{\circ}$. (d) AFM image. (e) X Cross sections above the trimer. (f) Calculated map of the absolute value of the field X component. (g), (h) zoom out AFM image for the $\theta=30^{\circ}$ case: (g) AFM image before exposure. (h) AFM image 
after exposure. (i),(j),(k) case of $\theta=-15^{\circ}$. (i) AFM image. (j) X Cross sections above the trimer. (k) Calculated map of the absolute value of the field $\mathrm{X}$ component. The white arrows represent the inplane projection of the incident wave vectors.

This near-field pattern is made of ultra-confined grazing propagating waves able to interfere with each other, reminding us what pioneers observed on metal nanostructures using first version of SNOM. ${ }^{68}$ A multiscale electromagnetic analysis is thus possible.

Figures $7(\mathrm{i}-\mathrm{k})$ show the $\theta=-15^{\circ}$ case. Again, the $\mathrm{C} 2$ nanogap is clearly turned on. It is worth noticing that cavity $\mathrm{C} 1$ is also activated: it presents a non-negligible topographical depletion that is about 2.2 times less deep than for nanogap C2. This partial activation is also shown in numerical simulation of $\mathrm{lE}_{\mathrm{x}} \mathrm{l}($ Figure $7(\mathrm{k}))$. This observed effect is in good agreement with the tendency revealed in Figure 6: tuning $\theta \square$ allows for an accurate tuning of the respective intensities of the different involved electromagnetic hot spots: $0^{\circ}$ does not enable any contrast between different hot spots, $-/+15^{\circ}$ leads to partial contrast, $-/+30^{\circ}$ allows for full hot spot selection.

In Figure 8 the obtained near-field data are analyzed in a more quantitative way. The volume of the nanometric topographical depletion measured at three different locations was plotted, using a $\ln$ - $\ln$ scale, as a function of corresponding calculated integrated intensity of the $\mathrm{x}, \mathrm{y}$ component of the electric field.

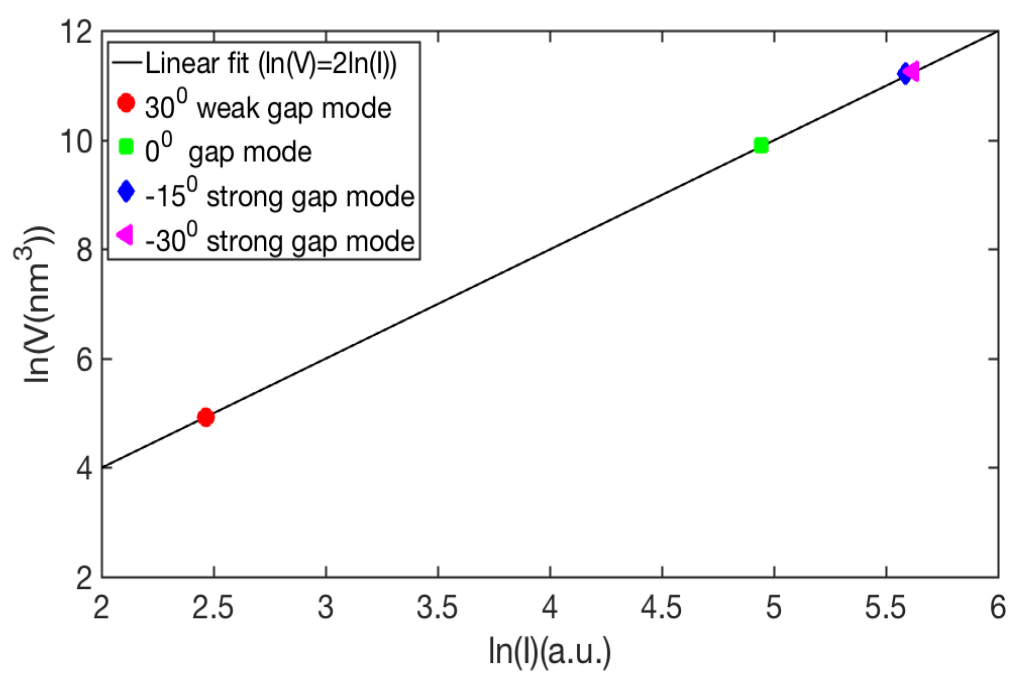

Figure 8 Analysis of the data. Measured Volume of nano topographical depletion in four cases as a function of calculated integrated intensity of the in-plane (x,y) component of the electric field (ln-ln plot). 
Four locations were considered: the $\mathrm{C} 2$ cavity in Figure 7(a), the $\mathrm{C} 2$ cavity in Figure 5(a), the C2 cavity in Figure 7(i), and the C2 cavity in Figure 7(d). We have made sure that these three situations i) correspond to the same exposure time and incident power, ii) do not correspond to any saturation of the matter migration, a phenomenon that can for example be suspected in Figure 5(e) where depletion depth $>30 \mathrm{~nm}$ were measured. The observed slope of 2 in Figure 8 is the signature of a nanoscale 2-photon absorption and opens up the route towards nearfield intensity quantification based on photo-induced molecular displacement: the measurement of the displaced volume of matter would lead to the assessment of the corresponding near-field intensity. Let us stress that these results are very preliminary in the sense that measuring a nano-volume in a precise way with an atomic force is still a challenge because it strongly depends on tip size and shape.

Finally, Figure 9 summarizes results obtained when considering (E1, C1, C2, E2) as a fourdigit nanosystem. Our motivation is to briefly discuss the possibility to address photonic bits for future data processing and storage. We noticed previously (Figures 5 and 7) that the extremities E1 and E2 are also susceptible to support electromagnetic hot spots, although with weaker intensities. As a consequence, migration of matter can also be observed at the extremities of the oligomer. E1, E2, C1, C2 hence constitute a 4-digit system. Figure 9 shows measured topographical depletion at the four digits in four cases corresponding to four incident angles: $0^{\circ}, 30^{\circ},-30^{\circ}, 15^{\circ}$ corresponding to Figures 5(a), 7(a), 7(d), 7(i), respectively. By applying a threshold, each value of depletion $(v d)$ can be associated to a binary state: 0 or 1. Here we take $v d \geq 4 \mathrm{~nm} \rightarrow$ state 1 ; vd $<4 \mathrm{~nm} \rightarrow$ state 0 , resulting in the four binary numbers shown in Figure 9.

With more complex plasmonic structures, any N-digit combination could thus be envisaged, remotely controlled through the angle of incidence and the polarization state of light.

Regarding future application of these switchable hot spots for information processing: azobenzene-containing polymer has only been used for probing electromagnetic hot spots, in 
order to demonstrate possible different 4-bit digits. This polymer and associated molecular movement are obviously not envisaged for nanophotonic information systems because 2photon induced movement in PAP is pretty slow and irreversible, which is not suitable for the information process.

For practical use in this context, bare trimers could be used and activated with fast switching of incident polarization and angle using for example optoelectronic modulator whose speed can nowadays reach $40 \mathrm{~GB} / \mathrm{s}$. The level of the $0 / 1$ optical state would be associated to the local intensity (instead of the local topographical depletion). The bridge between nanoscale hot spots and external modules could be ensured by integrated photonic guiding structure. Two examples of coupling between optical nanosources and guiding micro and nano structures are described in references. ${ }^{69,70}$

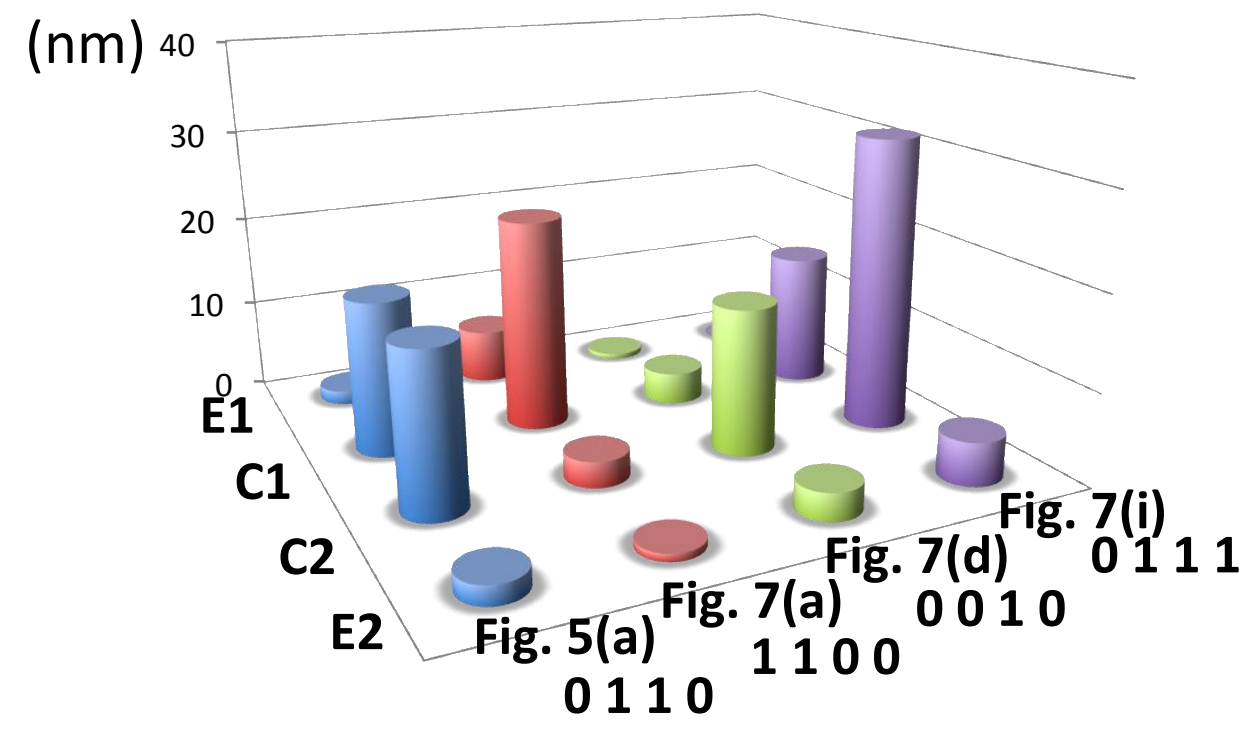

Figure 9. Near-field data summarized as 4-digit information: topographical depletion at different locations and corresponding binary 4-digit numbers.

Let us conclude by stressing the most important observations and achievements:

i) Hybrid modes in infrared 3-particle linear plasmonic oligomers were imaged with a spatial resolution of about $\lambda / 35$ (20 nm resolution, see "Method" section)

ii) A nanoscale and controlled spatial switch of the localized hot spots was 
demonstrated, as a signature of bonding and anti-bonding gap modes associated to radiant and subradiant modes of the trimer, by tuning of the incident angle and polarization, constituting a simple remote control.

iii) Two-photon absorption in azobenzene-containing photopolymer enables nanoscale photoinduced molecular displacement in the near-infrared. This displacement is triggered by the local plasmonic near-field

iv) A optical local field polarization contrast was observed (which would not possible with other methods like PEEM, EELS)

v) A preliminary quantification of the local intensity from the observed induced topography was reported.

vi) We also paid attention to the local field enhancement at the extremities of the trimer and discussed the plasmonic oligomer as a 4-bit nanophotonic information system.

These results provide a clear route towards near-field investigation of plasmonic antenna in the near-infrared.

\section{METHODS}

\section{Numerical Calculation}

The modeling of the scattering problem was performed using a diffracted field formulation of the Finite Element Method (FEM). The main difficulty relies here on the choice of the unknown field that has to satisfy a proper outgoing wave condition. A relevant approach is then to consider a planar reference structure composed of the planar embedding layer, superstrate and substrate only (the trimer is removed). The unknown field of our problem is then the difference between the total (reference and diffracted) field solutions of the diffraction problems when considering first the entire structure described in Figure 3 and, second, the reference planar structure. In that way, the problem is reduced to the calculation of the field radiated by the set of rods of the structure put in the reference field. Standard Cartesian PMLs adapted to the substrate, superstrate, and embedding layer were used to bound the computational domain and absorb the field radiated from the rods. The problem is solved using second order edge elements with a mesh size of $3 \mathrm{~nm}$ in and between the rods. Further details can be found in refenrence ${ }^{71}$. The model was implemented using the GNU softwares Gmsh and GetDP. ${ }^{72,73}$

\section{Photopolymer}

The azo dye molecule-containing polymer consists of pseudostilbene azobenzene molecules, disperse red 1(DR1), grafted to a side chain to polymethylmethacrylate (PMMA) in a 30\% molar ratio (PMMA-DR1). PMMA-DR1 is dissolved in 1,1,2-trichloroethane concentrated at 
$20 \mathrm{~g} / \mathrm{L}$, after stirring using magnetic stirrer for $5 \mathrm{~min}$ and filtering at room temperature, PMMA-DR1 solution is spin-coated on to the trimers with angular acceleration of $4000 \mathrm{rmp} / \mathrm{s}^{2}$ and angular speed of $3000 \mathrm{rmp} / \mathrm{s}$ for $30 \mathrm{~s}$. Agilent 5100 AFM measurements (tapping mode) showed that the polymer film is $45 \mathrm{~nm}$ thick on the substrate and about $10 \mathrm{~nm}$ thick above the top surface of nanoparticles. The same AFM is used for analysis of the photo-induced topography

\section{Exposure}

The $900 \mathrm{~nm}$ light issued from a Ti: Sa laser is linearly polarized, and focused by $10 \mathrm{X}$ nearinfrared objective lens with $18 \mathrm{~mm}$ working distance and 0.3 N.A. A landmark fabricated on the sample by Electron Beam Lithography was used to calibrate the position of laser spot and focus relative to the trimers. The entrance objective pupil is partially covered, resulting in a $100 \mu \mathrm{m}$ wide plane wave at the sample surface.

In the case of normal incidence, the sample is placed on the sample stage of microscope,

Irradiation of the sample is performed with a $\mathrm{Z}$ propagating plane wave (cf. figure 3 in manuscript). The power density is measured between the objective lens and the sample. In the case of oblique incidence, a sample stage is used rotate the $\mathrm{XZ}$ plane around the $\mathrm{Z}$ axis.

It should be pointed out that no modification of the shape of the gold trimers (due to thermal effects) has been observed, which is not surprising. In ref. ${ }^{74}$, thermal damage was observed from an average intensity of $350 \mu \mathrm{W}(\lambda=780 \mathrm{~nm}$, spot diameter $=1$ micron $)$, corresponding to about $35 \mathrm{~kW} / \mathrm{cm}^{2}$, which is much bigger than the power density used for our study (typically $300 \mathrm{~mW} / \mathrm{cm}^{2}$ ).

\section{Spatial resolution assessment}

Spatial resolution of our approach has been assessed. It is never easy to quantify a spatial resolution in scanning probe microscopy. In our case, this resolution depends on the tip shape and size, the hot spot size, and the response of the azobenzene molecules under local illumination. Let us consider Fig. 7. In Fig. 7 (c) and Fig. 7(f), theoretical hot spot lateral size at extremities E1 and E2 is about $20 \mathrm{~nm}$. Corresponding topographical depletion has a width in the 50-100 $\mathrm{nm}$ range, suggesting an effective spatial resolution in the $15-40 \mathrm{~nm}$ range. As another example, in Fig. 5(f), the Ey modulus map shows fours central hot spots having a 12 $\mathrm{nm}$ spatial expansion. The corresponding depletion is about $20 \mathrm{~nm}$, suggesting a resolution better than $10 \mathrm{~nm}$. As a result, we consider that the spatial resolution is about $20 \mathrm{~nm}$, which can be viewed as a mean value. It should be pointed out that $20 \mathrm{~nm}$ corresponds to the typical size of the extremity of the used silicon AFM tip (Nanosensors, PPP-NCLR-50, force constant: $21-98 \mathrm{~N} / \mathrm{m}$, resonance frequency: $146-236 \mathrm{kHz}$ )

\section{ACKNOWLEDGMENTS}

The authors thank the Agence Nationale de la Recherche (ANR), under Grants TWINS, NATO, HAPPLE and ACTIVE-NANOPHOT. R. B. thanks Ludovic Douillard for fruitful discussion about PEEM technique. Plasmonic samples were fabricated in the Nano'mat Platform (www.nanomat.eu). This work has been carried out in the context of the Labex ACTION project 


\section{REFERENCES}

(1) Maier, S. A Plasmonics: fundamental and applications, $7^{\text {th }}$ edition. Springer Verlag: New York, 2007.

(2) Advances in Plasmonics. 2016. Geddes, C. D., Ed.; Springer: New York, 2017.

(3) Liz-Marzán, L. M.; Murphy, C. J.; Wang, J. F. Nanoplasmonics. Chem. Soc. Rev. 2014,43, 3820-3822.

(4) Wei, H.; Xu, H. Plasmonics in composite nanostructures. Mater. Today 2014, 17, 372380.

(5) Lee, B.; Lee, I-M.; Kim, S.; Oh, D.-H; Hesselink, L. Review on subwavelength confinement of light with plasmonics. J. Mod. Opt. 2010, 57, 1479-1497.

(6) Savage, N. Photonics: Trick of the Light. Nature 2013, 495, S8-S9.

(7) Davis, T. J.; Gomez, D. E.; Roberts, A. Plasmonic Circuits for Manipulating Optical Information. Nanophotonics 2017, 6, 543-559.

(8) Ueno, K.; Misawa, H. Surface Plasmon-Enhanced Photochemical Reactions. J. Photochem. Photobiol. C Photochem. Rev. 2013, 15, 31-52.

(9) Linic, S.; Aslam, U.; Boerigter, C.; Morabito, M,. Photochemical Transformations on Plasmonic Metal Nanoparticles. Nat. Mater, 2015, 14, 567-576.

(10) Gray, S. K. Surface Plasmon-Enhanced Spectroscopy and Phtochemistry. Plasmonics, 2007, 2, 143-146.

(11) Deeb, C.; Ecoffet, C.; Bachelot, R.; Plain, J; Bouhelier, A.; Soppera, O. Plasmon-Based Free-Radical Photopolymerization: Effect of Diffusion on Nanolithography Processes. J. Am. Chem. Soc. 2011, 133, 10535-10542.

(12) Huang, J. S.; Yang, Y. T. Origin and Future of Plasmonic Optical Tweezers. Nanomaterials, 2015, 5, 1048-1065.

(13) Jensen, R. A.; Huang, I. C.; Chen, O.; Choy, J. T.; Bischof, T. S.; Loncar, M.; Bawendi, M. G. Optical Trapping and Two-Photon Excitation of Colloidal Quantum Dots Using Bowtie Apertures. ACS Photon. 2016, 3, 423-427.

(14) Mivelle, M.; Van Zanten, T. S.; Neumann, L.; Van Hulst, N. F.; Garcia-Parajo, M. F. Ultrabright Bowtie Nanoaperture Antenna Probes Studied by Single Molecule Fluorescence. Nano Lett. 2012, 12, 5972-5978.

(15) Juan, M. L.; Righini, M.; Quidant, R. Plasmon Nano-Optical Tweezers. Nat. Photonics 2011, 5, 349-356.

(16) Kauranen, M.; Zayats, A. V. Non linear Plasmonics. Nat. Photonics. 2012, 6, 737-748.

(17) Wurtz, G. A.; Pollard, R.; Hendren, W.; Wiederrecht, G. P.; Gosztola, D. J.; Podolskiy, V. A.; Zayats, A. V. Designed Ultrafast Optical Nonlinearity in a Plasmonic Nanorod Metamaterial Enhanved by Nonlocality. Nat. Nanotechnol. 2011, 6, 107-111.

(18) Butet, J.; Brevet, P.-F.; Martin, O. J. F. Optical Second Harmonic Generation in Plasmonic Nanostructures: From Fundamental Principles to Advanced Applications. ACS Nano 2015, 9, 10545-10562.

(19) Muehlethaler, C.; Leona, M.; Lombardi, R. Review of Surface Enhanced Raman Scattering Applications in Forensic Science. Anal. Chem. 2016, 88, 152-169.

(20) Schlucker, S. Surface-Enhanced Raman Spectroscopy: Concepts and Chemical Applications. Angew. Chem. Int. Ed. 2014, 53, 2-42.

(21) Rumyantseva, A.; Kostcheev, S.; Adam, P. M.; Gaponenko, S. V.; Vaschenko, S. V.; Kulakovich, O. S.; Ramanenka, A. A.; Guzatov, D. V.; Korbutyak, D.; Dzhagan, V.; Stroyuk, A.; Shvalagin, V. Nonresonant Surface-Enhanced Raman Scattering of Zno Quantum Dots with Au and Ag Nanoparticles. ACS Nano 2013, 7, 3420-3426.

(22) Stockman, M. I. Spasers Explained. Nat. Photonics 2008, 2, 327-329. 
(23) Fort, E.; Gresillon, S. Surface Enhanced Fluorescence. J. Phys. D: Appl. Phys. 2008, 41, 1-31.

(24) Yang, A.; Hoang, T. B.; Dridi, M.; Deeb, C; Mikkelsen, M. H.; Schatz, G. C.; Odom, T. W. Real-Time Tunable Lasing from Plasmonic Nanocavity Arrays. Nat. Commun. 2015, 6, 6939.

(25) Zhou, X.; Wenger, J.; Viscomi, F. N.; Cunff, L. L.; Beal, J.; Kochtcheev, S.; Yang, X. Wiederrecht, G. P.; Colas des Francs, G.; Bisht, A. S.; Jradi, S.; Caputo, R.; Demir, H. V.; Schaller, R. D.; Plain, J.; Vial, A.; Sun, X. W.; Bachelot, R. Two-Color Single Hybrid Plasmonic Nanoemitters with Real Time Switchable Dominant Emission Wavelength. Nano Lett. 2015, 15, 7458-7466.

(26) Schietinger, S.; Barth, M.; Aichele, T.; Benson, O. Plasmon-Enhanced Single Photon Emission from a Nanoassembled Metal-Diamond Hybrid Structure at Room Temperature. Nano lett. 2009, 9 , 1694-1698.

(27) Viste, P.; Plain, J.; Jaffiol, R.; Vial, A.; Adam, P. M.; Royer, P. Enhancement and Quenching Regimes in Metal-Semiconductor Hybrid Optical Nanosources. ACS Nano 2010, $4,759-764$.

(28) Celebrano, M.; Lettow, R.; Kukura, P.; Agio, M.; Renn, A.; Gotzinger, S.; Sandoghdar, V. Efficient Coupling of Single Photons to Single Plasmons. Opt. Express 2010, 18, 1382913835 .

(29) Brongersma, M. L.; Halas, N. J.; Nordlander, P. Plasmon-Induced Hot Carrier Science and Technology. Nat. Nanotechnol. 2015, 10, 25-34.

(30) Sundararaman, R.; Narang, P.; Jermyn, A. S.; Goddard III, W. A.; Atwater, H. A. Theoretical Predictions for Hot-Carrier Generation from Surface Plasmon Decay. Nat. Commun. 2014, 5, 5788.

(31) Harutyunyan, H.; Martinson, A. B. F.; Rosenmann, D.; Khorashad, L. K.; Besteiro, L. V.; Govorov, A. O.; Wiederrecht, G. P. Anomalous Ultrafast Dynamics of Hot Plasmonic Electrons in Nanostructures with Hot Spots. Nat. Nanotechnol. 2015, 10, 770-774.

(32) Hentschel, M.; Dregely, D.; Vogelgesang, R.; Giessen, H; Liu, N. Plasmonic Oligomers: The Role of Individual Particles in Collective Behavior. ACS Nano 2011, 5, 2042-2050.

(33) Pasterilli, F.; Bidault, Morterell, J.; Bonod, N. Self-Assembled Plasmonic Oligomers for Organic Photovoltaics. Adv. Opt. Mat, 2014, 2, 171-175.

(34) Zhao, J.; Frank, B.; Burger, S.; Giessen, H. Large-Area High-Quality Plasmonic Oligomers Fabricated by Angle-Controlled Colloidal Nanolithography. ACS Nano 2011, 5, 9009-9016.

(35) Knebl, D.; Hörl, A.; Trügler, A.; Kern, J.; Krenn, j. R.; Puschnig, P.; Hohenester, U. Gap plasmonics of silver nanocube dimers. Phys. Rev. B 2016, 93, 081405(R).

(36) Roller, E.-M.; Besteiro, L. V.; Pupp, C.; Khosravi Khorashad, L. Govorov, A. O.; Liedl, T. Hotspot-mediated non-dissipative and ultrafast plasmon passage. Nat. Phys. 2017, $13,761-765$.

(37) Zohar, N. Chuntonov, L.; Haran, G. The simplest plasmonic molecules: Metal nanoparticle dimers and trimers. J. Photochem. Photobio C: Photochem. Rev. 2014, 21, 2639.

(38) Funston A. M.; Davis, T. J.; Novo, C.; Mulvaney, P. Coupling modes of gold trimer superstructures. Phil. Trans. R. Soc. A 2011, 369, 3472-348.

(39) Volpe, G.; Cherukulappurath, S.; Parramon, R. J.; Molina-Terriza, G.; Quidant, R. Controlling the optical near field of nanoantennas with spatial phase-shaped beams. Nano Lett. 2009, 9, 3608-3611.

(40) Devilez, A.; Stout, B.; Bonod, N. Mode-balancing far-field control of light localization in nanoantennas. Phys. Rev. B. 2010, 81, 245128. 
(41) Alonso-González, P.; Albella, P.; Golmar, F. ; Arzubiaga, L.; Casanova, F.; Hueso, L. E.; Aizpurua, J.; Hillenbrand, R. Visualizing the near-field coupling and interference of bonding and anti-bonding modes in infrared dimer nanoantennas. Opt. Expr. 2013, 21, 12701280 .

(42) Fisher, U. C. Scanning near-field optical microscopy (Chap. 7). In Scanning Probe Microscopy: Analytical Methods Roland Wiesendanger Springer Science \& Business Media, 14 mars 2013 - 216 pages

(43) Greffet. J.-J.; Carminati,R. Image formation in near-field optics. Prog. Surf. Sci. 1997, $56,133-237$.

(44) van Hulst, N. F.; Segerink, F. B.; Achten, F.; Böger, B. Evanescent-field optical microscopy: effects of polarization, tip shape and radiative waves. Ultramicroscopy 1992, 42, 416-421

(45) Gomez, L.; Bachelot, R.; Bouhelier, A.; Wiederrecht, G. P.; Chang, S.-H.; Gray, S. K.; Hua, F.; Jeon, S.; Rogers, J. A.; Castro, M. E. Apertureless scanning near-field optical microscopy: a comparison between homodyne and heterodyne approaches. J. Opt. Soc. Am. B. 2006, 23, 823-833.

(46) Douillard, L.; Charra, F.; Korczak, Z.; Bachelot, R.; Kostcheev, S.; Lerondel, G.; Adam, P.-M.; Royer; P. Short Range Plasmon Resona- tors Probed by Photoemission Electron Microscopy. Nano Lett. 2008, 8, 935-940.

(47) Hrelescu, C.; Sau, T. K.; Rogach, A. L.; Jackel, F.; Laurent, G.; Douillard, L.; Charra, F. Selective Excitation of Individual Plasmonic Hotspots at the Tips of Single Gold Nanostars. Nano Lett. 2011, 11, 402-407.

(48) Word, R. C.; Fitzgerald, J. P. S.; Könenkamp, R. Direct Coupling of Photonic Modes and Surface Plasmon Polaritons Observed in 2- Photon PEEM. Opt. Express. 2013, 21, 30507-30520.

(49) Koh, A. L.; Bao, K.; Khan, I.; Smith, W. E.; Kothletner, G.; Nordlander, P.; Maier, S. A.; McComb, D. W. Electron Energy-Loss Spectroscopy Silver Nanoparticles and Dimers: Influence of Beam Damage and Mapping of Dark Modes. ACS Nano 2009, 3, 3015-3022.

(50) Koh, A. L.; Fernandez-Dominguez, A. I.; McComb, D. W.; Maier, S. A.; Yang, J. K. W. High-Resolution Mapping of Electron-Beam-Excited Plasmon Modes in Lithographically Defined Gold Nanostructures. Nano Lett. 2011, 11, 1323-1330.

(51) Colliex, C.; Kociak, M.; Stéphan, O. Electron Energy Loss Spectroscopy imaging of surface plasmons at the nanometer scale. Ultramicroscopy 2016, 162, A1-A24.

(52) Martin, J.; Kociak, M.; Mahfoud, Z.; Proust J.; Gérard, D.; Plain, J. High-Resolution Imaging and Spectroscopy of Multipolar Plasmonic Resonances in Aluminum Nanoantennas Nano Lett. 2014, 14, 5517-5523.

(53) Hörl; A.; Trügler, A.; Hohenester, H. Tomography of Particle Plasmon Fields from Electron Energy Loss Spectroscopy. Phys. Rev. Lett. 2013, 111, 076801.

(54) Hohenester, U.; Ditlbacher, H.; Krenn, J. R. Electon Energy Loss spectra of plasmonic nanoparticles. Phys. Rev. Lett. 2009, 103, 106801.

(55) Frimmer, M.; Coenen, T.; Koenderink, A. F. Signature of a fano resonance in plasmonic metamolecule' local density of optical states. Phys. Rev. Lett. 2012, 108, 077404.

(56) Mirin, N. A., Bao, K.; Nordlander, P.; Fano Resonances in Plasmonic Nanoparticle Aggregates. J. Phys. Chem. A 2009, 113, 4028-4034.

(57) Hubert, C.; Rumyantseva, A.; Lerondel, G.; Grand, J.; Kotscheev, S.; Billot, L.; Vial, A.; Bachelot, R.; Royer, P.; Chang, S.; Gray, S. K.; Wiederrecht, G. P.; Schatz, G. C. NearField Photochemical Imaging of Noble Metal Nanostructures. Nano Lett. 2005, 5, 615-619.

(58) Hubert, C.; Bachelot, R.; Plain, J.; Kostcheev, S.; Lerondel, G.; Juan, M.; Royer, P.; Zou, S.; Schatz, G. C.; Wiederrecht, G. C.; and Gray, S. K. Near-Field Polarization Effects in Molecular-Motion-Induced Photochemical Imaging J. Phys. Chem. C 2008, 112, 4111-4116. 
(59) Juan, M.; Plain, J.; Bachelot, R.; Vial, A.; Royer, P.; Gray, S. K.; Montgomery, J.; and Wiederrecht, G. P. Plasmonic Electromagnetic Hot Spots Temporally Addressed by Photoinduced Molecular Displacement $\dagger$. J. Phys. Chem A 2009, 113, 4647-4651.

(60) Juan, M. L. ; Plain, J. ; Bachelot, R. ; Royer, P. ; Gray, S. K. ; Wiederrecht, G. P. Multiscale Model for Photoinduced Molecular Motion in Azo Polymers. ACS Nano 2009, 3, 1573-1579.

(61) Juan, M. ; Plain, J. ; Bachelot, R. ; Royer, P. ; Gray, S. ; et Wiederrecht, G. SelfConsistent Model of Light-Induced Molecular Motion Around Metallic Nanostructures. $J$. Phys. Chem. Lett. 2010, 1, 2228-2232.

(62) Haggui, M. ; Dridi, M. ; Plain, J. ; Marguet, S. ; Henri, P., Schatz, G. ; Wiederrecht, G. ; Gray, S. ; Bachelot, R. Spatial Confinement of Electromagnetic Hot and Cold Spots in Gold Nanocubes. ACS Nano 2012, 6, 1299-1307.

(63) Plain, J. ; Wiederrecht, G.P., Gray, S.K. ; Royer, P. ; Bachelot, R. Multiscale optical imaging of complex fields based on the use of azobenzene nanomotors. J. Phys. Chem. Lett. 2013, 4, 2124-2132.

(64) Galarreta, B.; I. Rupar, I.; Young, A.; Lagugné-Labarthet, F. Mapping Hot-Spots in Hexagonal Arrays of Metallic Nanotriangles. J. Phys. Chem. C. 2011, 115, 15318-15323.

(65) Teulle, A.; Sanchot, A.; Ishow, E.; Sharma, J.; Dujardin, E. Photochemical Mapping of the Multimodal Plasmonic Response of 2D Gold Crystals. Phys. Chem. C. 2017, 121, 1590815914.

(66) Lefin, P.; Fiorini, C.; Nunzi, J. Anisotropy of the photo-induced translation diffusion of azobenzene dyes in polymer matrices. Pure Appl. Opt. 1998, 7, 71-82.

(67) Ishitobi, H.; Shoji, S.; Hiramatsu, T.; Sun, H.-B.; Sekkat, Z.; Kawata, S. Two-photon induced polymer nanomovement. Opt. Express 2008, 16, 14106-14114.

(68) Krenn, J. R.; Weeber, J. C.; Dereux, A.; Bourillot, E.; Goudonnet, J. P.; Schider, B.; Leitner, A.; Aussenegg, F. R.; Girard, C. Direct observation of localized surface plasmon coupling. Phys. Rev. B 1999, 60, 5029-5033.

(69) Weeber, J.-C.; Hammani, K.; Colas-des-Francs, G.; Bouhelier, A.; Arocas, J.; Kumar, A.; Eloi, F.; Buil, S.; Quelín, X.; Hermier, J.-P.; Nasilowski, M.; Dubertre, B. Colloidal Quantum Dot Integrated Light Sources for Plasmon Mediated Photonic Waveguide Excitation. ACS Photon. 2016, 3, 844-852.

(70) Luo, Y.; Chamanzar, M.; Apuzzo, A.; Salas-Montiel, R.; Nguyen, K. N.; Blaize, S.; Adibi, A. On-Chip Hybrid Photonic-Plasmonic Light Concentrator for Nanofocusing in an Integrated Silicon Photonics Platform. Nano Lett. 2015, 15, 849-856.

(71) Demésy, G. ; Zolla, F. ; Nicolet, A.; Commandré, M. All-purpose finite element formulation for arbitrarily shaped crossed-gratings embedded in a multilayered stack. J. Opt. Soc. Am. 2010, A 27, 878-889.

(72) Geuzaine, C.; Remacle, J. F. Gmsh: A 3-D finite element mesh generator with built-in pre-and post-processing facilities. International journal for numerical methods in engineering 2009, 79, 1309-1331.

(73) Dular, P.; Geuzaine, C.; Henrotte, F.; Legros, W. A general environment for the treatment of discrete problems and its application to the finite element method. IEEE Transactions on Magnetics 1998, 34, 3395-3398.

(74) Bouhelier, A.; Bachelot, R.; Lerondel, G.; Kostcheev, S.; Royer, P.; Wiederrecht, G. P. Surface Plasmon Characteristics of Tunable Photoluminescence in Single Gold Nanorods. Phys. Rev. Lett. 2005, 95, 267405. 


\section{TOC GRAPHIC}

